\title{
STORM WATER MANAGEMENT AT ARLANDA AIRPORT
}

\author{
Lars Kylefors \\ Vatten och Samhällsteknik, Kalmar, Sweden
}

\begin{abstract}
Vatten och Samhällsteknik has since 1985 been deeply engaged in developing purification systems for polluted waters by the use of constructed wetlands. Such wetlands can be designed in extensive or intensive way depending on the local situation (polluting parameters) and environmentally demands.

Example of pollutions can come from

- Agriculture, especially nitrogen and phosphorus

- Storm water from cities, especially heavy metals and oil rest products

- Leachate from landfills, especially organic substances, nitrogen and heavy metals

- Storm water from Airports, especially organic substances and heavy metals
\end{abstract}

The possible purification in a constructed wetland of such pollutions can be based on a lot of different processes depending of the design, such as:

- Aeration

- Sedimentation

- Growth and harvest of water plants/algae

- Filtration

- Micro organisms

This abstract will focus on storm water from airports and the case Arlanda Airport.

The specific pollution at airports in Sweden and other northern countries is the result of winter operation. At most northern airports formiat is used to clear the runways from ice. Snow will mainly be removed in mechanic ways and also warm sand can be part of a winter operation. In some special occasions and at some airports also urea (concentrated ammonia) might be used. For de-icing the aircrafts different sorts of glycol normally is used. Both formiat and glycol are organic substances, very effective to melt the ice, but they will be broken down rather quickly and during this process demand oxygen. The result in a recipient will be that existing oxygen in the water will end and living fauna rapidly die.

At Arlanda Airport the storm water management together with the total operation has been proven by the Environmental Court (now in a higher court). Swedavia has promised and already built four plants for managing of the storm water pollutions, especially from the 
runways and terminal areas. Kättstabäckens dagvattenanläggning has been in use for some years and Halmsjöbäckens dagvattenanläggning is now ready to use.

Halmsjöbäckens dagvattenanläggning, see fig 1, will take care of the storm water mainly from runway 3. The Environmental Court has decided that the results from the four plants shall be tested and optimised during a test period of three years.

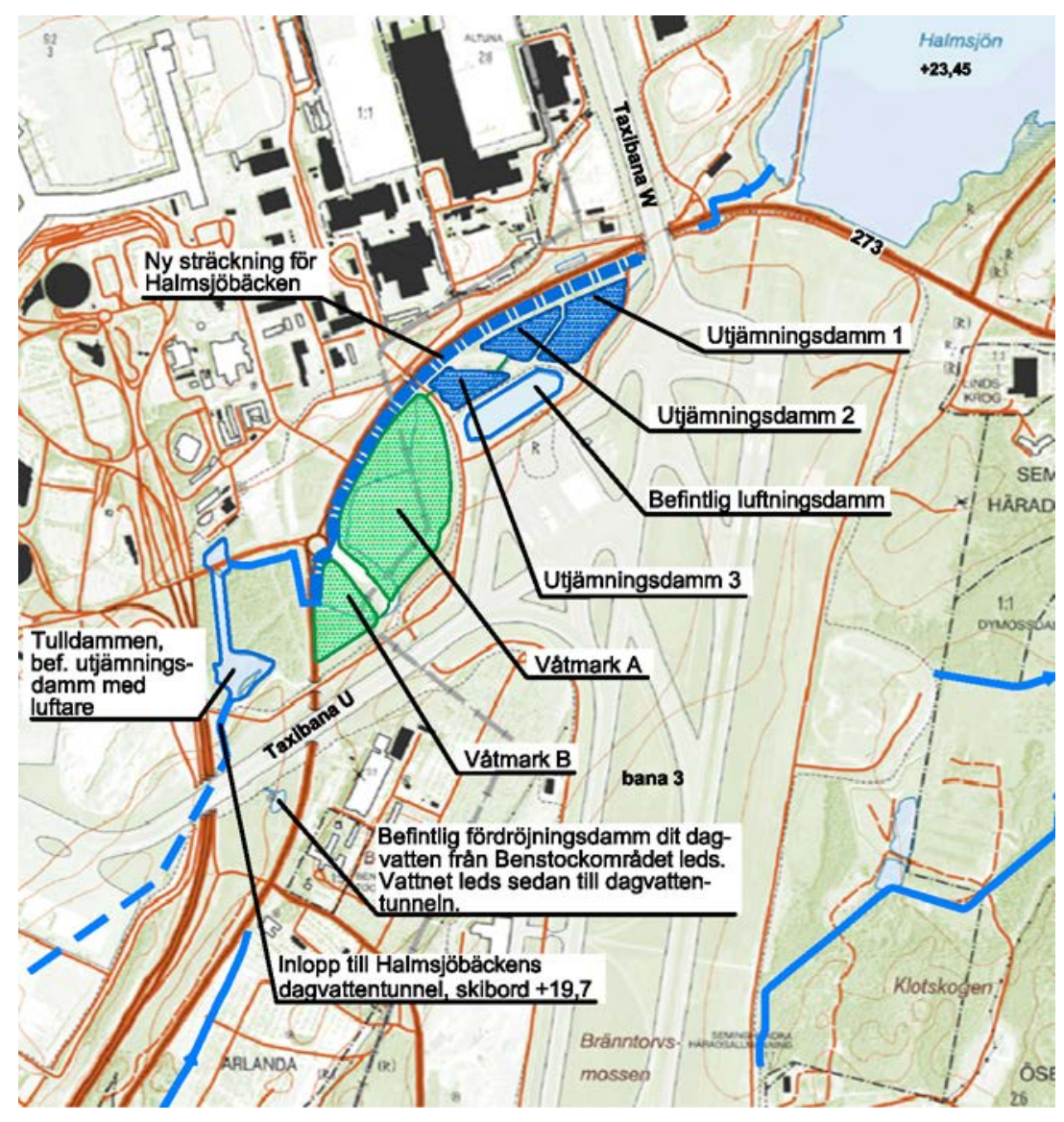

Fig 1. Halmsjöbäckens dagvattenanläggning

Halmsjöbäckens dagvattenanläggning is constructed for different processes, mainly:

- Dams as compensation from winter to summer

- Aeration to continuously add oxygen

- Sedimentation of oxides of metals and organically fixed phosphorous

- Denitrification 
The demands from the Environmental Court are:

- Organic substances shall be decreased from more than $150 \mathrm{mg} / \mathrm{l}$ to about TOC $16 \mathrm{mg} / \mathrm{l}$

- $\quad$ The oxygen content in the recipient down streams shall be at least $5 \mathrm{mg} / \mathrm{l}$

- The heavy metals shall be less than what is said in EU:s framework of waters

- The nitrogen and phosphorous content shall be decreased to make it possible to fulfil the ecological goals for the recipient

Some of the results from monitoring the treatment of storm water are illustrated in the following figures.

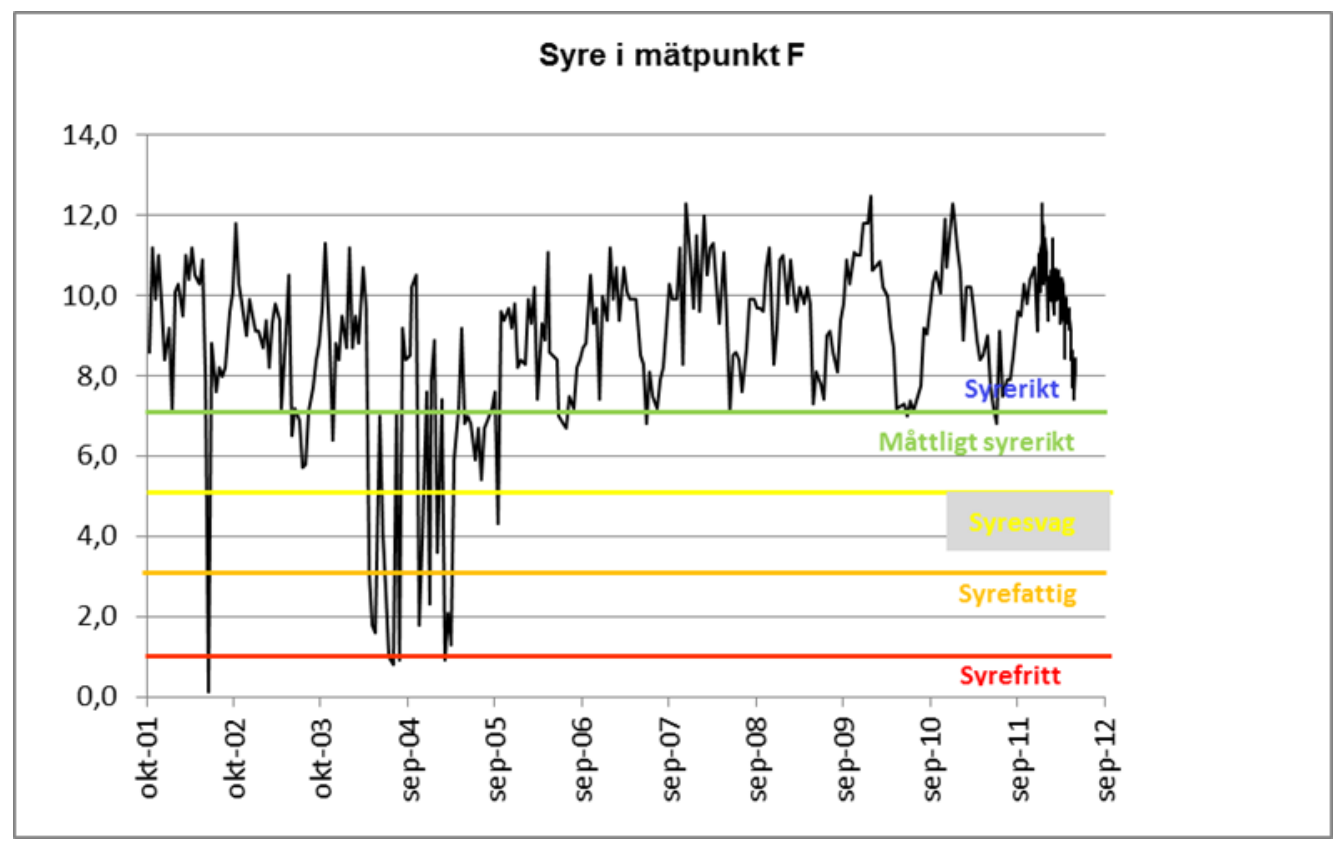

Concentration of oxygen at section F. 
Linnaeus ECO-TECH '14,

Kalmar, Sweden, November 24-26, 2014

\section{Concentration of heavy metals at section F}

\begin{tabular}{|c|c|c|c|c|c|c|c|c|c|c|c|c|}
\hline Datum & $\begin{array}{l}\mathrm{Pb} \\
\mu \mathrm{g} / \mathrm{l}\end{array}$ & $\begin{array}{l}\mathrm{Pb} * \\
\mu \mathrm{g} / \mathrm{I}\end{array}$ & $\begin{array}{l}\mathrm{Cd} \\
\mu \mathrm{g} / \mathrm{I}\end{array}$ & $\begin{array}{l}\mathrm{Cd}^{*} \\
\mu \mathrm{g} / \mathrm{l}\end{array}$ & $\begin{array}{r}\mathrm{Cu} \\
\mu \mathrm{g} / \\
\end{array}$ & $\begin{array}{l}\mathrm{Cu}^{*} \\
\mu \mathrm{g} / \mathrm{I}\end{array}$ & $\begin{array}{l}\mathrm{Cr} \\
\mu \mathrm{g} / \mathrm{l}\end{array}$ & $\begin{array}{l}\mathrm{Cr}{ }^{*} \\
\mu \mathrm{g} / \mathrm{I}\end{array}$ & $\begin{array}{r}\mathrm{Ni} \\
\mu \mathrm{g} / \mathrm{I}\end{array}$ & $\begin{array}{l}\mathrm{Ni}^{*} \\
\mu \mathrm{g} / \\
\end{array}$ & $\begin{array}{l}\mathrm{Zn} \\
\mu \mathrm{g} / \mathrm{I}\end{array}$ & $\begin{array}{l}\mathrm{Zn}{ }^{*} \\
\mu \mathrm{g} / \mathrm{I}\end{array}$ \\
\hline 090416 & $<1,2$ & $<0,5$ & $<0,12$ & $<0,05$ & 6,0 & 4,2 & 4,7 & 1,00 & 6,3 & 3,9 & 20 & 6 \\
\hline 090514 & $<0,5$ & & $<0,12$ & & 4,4 & & 1,4 & & 4,1 & & 10 & \\
\hline 090609 & 0,60 & $<0,5$ & $<0,05$ & $<0,05$ & 4,8 & 4,5 & 1,6 & 1,50 & 2,7 & 2,3 & 9 & $<5$ \\
\hline 090723 & $<1,2$ & & $<0,12$ & & 3,0 & & 1,9 & & 5,6 & & 54 & \\
\hline 090817 & $<1,2$ & $<0,5$ & $<0,12$ & $<0,05$ & 5,9 & 3,9 & $<1,2$ & 0,70 & 5,5 & 3,7 & 12 & $<5$ \\
\hline 090918 & 1,80 & & $<0,05$ & & 3,0 & & 1,8 & & 4,9 & & 11 & \\
\hline 091014 & $<3,1$ & $<0,5$ & $<0,05$ & $<0,05$ & 7,2 & 3,8 & 3,4 & 1,80 & 5,2 & 4,9 & 33 & 7 \\
\hline 091111 & $<1,2$ & & $<0,12$ & & 6,2 & & 1,8 & & 5,9 & & 13 & \\
\hline 091208 & $<1,2$ & $<0,5$ & $<0,12$ & $<0,05$ & 6,5 & 4,1 & 2,8 & 2,70 & 5,1 & 5 & 17 & 8 \\
\hline 100108 & $<1,2$ & & $<0,12$ & & 4,0 & & 6,7 & & 4,0 & & $<12$ & \\
\hline 100217 & $<1,2$ & $<0,5$ & $<0,12$ & $<0,05$ & 3,4 & 1,8 & 4,2 & $7,90^{\star *}$ & 4,0 & 3 & $<12$ & $<5$ \\
\hline 100317 & $<1,2$ & & $<0,12$ & & 5,4 & & 1,7 & & 4,7 & & $<12$ & \\
\hline 100415 & $<1,2$ & $<0,5$ & $<0,12$ & $<0,05$ & 5,7 & 4,7 & 1,5 & $<0,5$ & 4,7 & 3,5 & $<12$ & 8 \\
\hline 100512 & $<1,2$ & & $<0,12$ & & 3,1 & & 1,5 & & 3,1 & & $<12$ & \\
\hline 100609 & $<1,2$ & $<0,5$ & $<0,12$ & $<0,05$ & 3,1 & 1,7 & 4,2 & 2,00 & 3,6 & 2,2 & $<12$ & $<5$ \\
\hline 100722 & 1,70 & & $<0,12$ & & 5,4 & & 4,1 & & 3,0 & & 21 & \\
\hline 100818 & $<1,2$ & $<0,5$ & $<0,12$ & $<0,05$ & 5,7 & 3,0 & 2,3 & $13^{\star *}$ & 3,4 & 2,6 & $<12$ & $<5$ \\
\hline 100915 & $<1,2$ & & $<0,12$ & & 4,8 & & 2,1 & & 3,1 & & $<12$ & \\
\hline medel hyd. år 09/10 & & & & & 5,0 & 3,2 & 3,0 & 1,8 & 4,2 & 3,5 & 15 & 6 \\
\hline max hyd. år 09/10 & & & & & 7,2 & 4,7 & 6,7 & 2,7 & 5,9 & 5,0 & 33 & 8 \\
\hline $\begin{array}{l}101013 \\
101109\end{array}$ & $\begin{array}{l}<1,2 \\
<1,2\end{array}$ & & $\begin{array}{l}<0,12 \\
<0,12\end{array}$ & & $\begin{array}{l}3,3 \\
4,0\end{array}$ & 2,0 & $\begin{array}{l}1,8 \\
1,7\end{array}$ & $<0,5$ & $\begin{array}{l}5,8 \\
3,8\end{array}$ & $10^{\times x}$ & $\begin{array}{l}<12 \\
<12\end{array}$ & 5 \\
\hline 101208 & $<1,2$ & $<0,5$ & $<0,12$ & $<0,05$ & 3,3 & 2,6 & 2,0 & 2,20 & 3,5 & 2,8 & $<12$ & 6 \\
\hline 110105 & $<1,2$ & & $<0,12$ & & 3,2 & & 1,8 & & 2,7 & & 14 & \\
\hline 110216 & $<1,2$ & $<0,5$ & $<0,12$ & $<0,05$ & 3,1 & 1,3 & 2,3 & 1,70 & 2,6 & 2,1 & 66 & 6 \\
\hline 110315 & $<1,2$ & & $<0,12$ & & 7,9 & & 2,4 & & 4,1 & & $<12$ & \\
\hline 110413 & $<1,2$ & $<1,2$ & $<0,12$ & $<0,12$ & 5,9 & 4,3 & 2,9 & $<1,2$ & 3,4 & 2,3 & 22 & 17 \\
\hline 110511 & $<1,2$ & & $<0,12$ & & 3,5 & & 1,4 & & 2,1 & & 18 & \\
\hline 110609 & 1,80 & $<1,2$ & $<0,12$ & $<0,12$ & 4,5 & 2,3 & 3,2 & 1,6 & 3,5 & 2,6 & 14 & 18 \\
\hline 110715 & 1,40 & & 0,02 & & 1,8 & & 0,6 & & 3,3 & & 7 & \\
\hline $\begin{array}{l}110817 \\
110915\end{array}$ & 0,31 & $<1,3$ & $\begin{array}{l}0,01 \\
0\end{array}$ & $<0,13$ & 3,0 & 2,9 & $\begin{array}{l}0,6 \\
07\end{array}$ & $<1,3$ & $\begin{array}{l}2,6 \\
42\end{array}$ & $<1,3$ & $\begin{array}{l}6 \\
8\end{array}$ & $<13$ \\
\hline medel hyd. år 10/11 & & & & & $\overline{4,0}$ & 2,6 & $\overline{1,8}$ & 1,4 & $\overline{3,5}$ & 2,2 & $\overline{17}$ & 11 \\
\hline max hyd. år 10/11 & & & & & 7,9 & 4,3 & 3,2 & 2,2 & 5,8 & 2,8 & 66 & 18 \\
\hline 111011 & & 0,03 & & $<0,01$ & & 3,4 & & 0,15 & & 3,2 & & 4 \\
\hline 111026 & & 0,02 & & $<0,01$ & & 3,5 & & 0,17 & & 3,6 & & 3 \\
\hline 111108 & & 0,02 & & $<0,01$ & & 7,3 & & 0,17 & & 3,8 & & 4 \\
\hline 111123 & & 0,02 & & 0,01 & & 4,8 & & 0,20 & & 4,1 & & 3 \\
\hline 111207 & & 0,03 & & $<0,01$ & & 5,2 & & 0,20 & & 3,3 & & 3 \\
\hline 111222 & & 0,05 & & 0,02 & & 3,8 & & 0,24 & & 3,3 & & 5 \\
\hline & & $<0,2$ & & $<0,02$ & & 3,9 & & $<0,5$ & & 3,9 & & $<5$ \\
\hline 120229 & & 0,04 & & 0,03 & & 3,1 & & 0,81 & & 9,9 & & 10 \\
\hline 120330 & & 0,08 & & 0,02 & & 3,0 & & 0,32 & & 4 & & 4 \\
\hline 120502 & & 0,11 & & 0,01 & & 3,1 & & 0,34 & & 3,5 & & 3 \\
\hline 120607 & & 0,06 & & $<0,01$ & & 2,9 & & 0,27 & & 3,2 & & 3 \\
\hline 120702 & & 0,05 & & $<0,01$ & & 2,9 & & 0,22 & & 3,4 & & 2 \\
\hline 120801 & & 0,06 & & $<0,01$ & & 2,6 & & 0,17 & & 3,4 & & 2 \\
\hline 120912 & & 0,06 & & $<0,01$ & & 3,1 & & 0,13 & & 2,7 & & 1 \\
\hline medel hyd. år 11/12 & & & & & & 3,8 & & 0,35 & & 4,3 & & 4 \\
\hline max hyd. ảr $11 / 12$ & & & & & & 3,9 & & 0,81 & & 9,9 & & 10 \\
\hline
\end{tabular}

Tillståndsklassning sjöar och vattendrag. NV rapport 4913***

\begin{tabular}{|l|c|c|c|c|c|c|c|c|c|c|c|c|}
\hline Mkt låg & $<0,2$ & $<0,2$ & $<0,01$ & $<0,01$ & $<0,5$ & $<0,5$ & $<3$ & $<3$ & $<0,7$ & $<0,7$ & $<5$ & $<5$ \\
Låg & 0,2 & 0,2 & 0,01 & 0,01 & 0,5 & 0,5 & 3 & 3 & 0,7 & 0,7 & 5 & 5 \\
Måttlig & 1 & 1 & 0,1 & 0,1 & 3 & 3 & 5 & 5 & 15 & 15 & 20 & 20 \\
Hög & 3 & 3 & 0,3 & 0,3 & 9 & 9 & 15 & 15 & 45 & 45 & 60 & 60 \\
\hline Mkt hög & 15 & 15 & 1,5 & 1,5 & 45 & 45 & 75 & 75 & 225 & 225 & 300 & 300 \\
\hline
\end{tabular}

Tillståndsklassning förorenat ytvatten. NV rapport 4918

\begin{tabular}{|l|c|c|c|c|c|c|c|c|c|c|c|c|}
\hline Mindre allvarligt & 3 & & 0,3 & & 9 & & 15 & & 45 & & 60 & \\
\hline
\end{tabular}

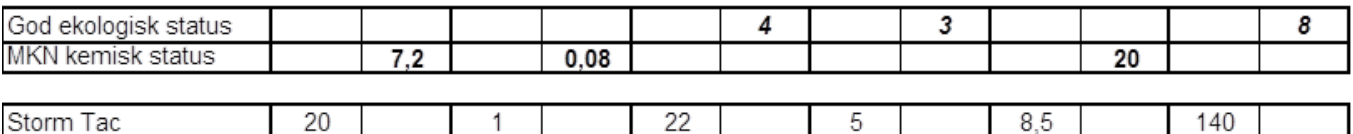

* Filtrerat prov

** Troligen någon form av analysfel, ej medtagen i medel- och maxvärdet eller i jmf med bedömningsvärdet.

*** Bedömningsvärdena är satta för ofiltrerade prov. 


\section{Load of tot-P at different sections}

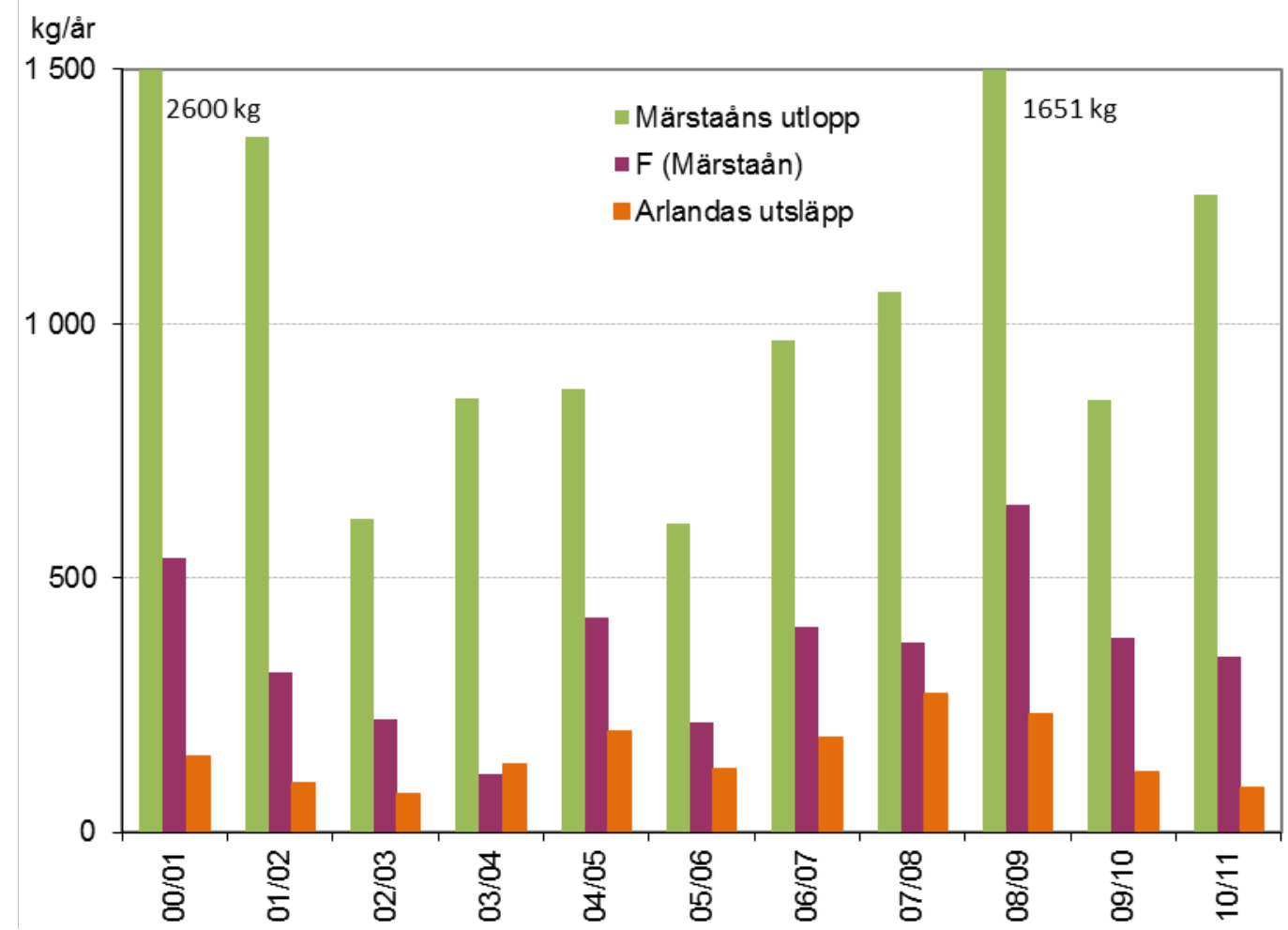

So far the results from Kättstabäckens dagvattenanläggning (KDA), that has been in operation for some year, show that the expectations are possible to fulfil. 